\title{
The core-wing anomaly of cool Ap stars ${ }^{\star}$
}

\section{Abnormal Balmer Profiles}

\author{
C. R. Cowley ${ }^{1}$, S. Hubrig ${ }^{2, \star \star}$, T. A. Ryabchikova ${ }^{4,6}$, G. Mathys ${ }^{3}$, N. Piskunov ${ }^{5}$, and P. Mittermayer ${ }^{6}$ \\ 1 Department of Astronomy, University of Michigan, David Dennison Building, Ann Arbor, Michigan 48109-1090, \\ USA \\ 2 Astrophysikalisches Institut Potsdam, An der Sternwarte 16, 14482 Potsdam, Germany \\ e-mail: shubrig@aip.de \\ 3 European Southern Observatory, Casilla 19001, Santiago 19, Chile \\ e-mail: gmathys@eso.org \\ 4 Institute of Astronomy, Russian Academy of Sciences, Pyatnitskaya 48, 109017 Moscow, Russia \\ e-mail: ryabchik@inasan.rssi.ru \\ ${ }^{5}$ Uppsala Astronomical Observatory, Box 515, 751, 20 Uppsala, Sweden \\ e-mail: piskunov@astro.uu.se \\ ${ }^{6}$ Institute for Astronomy, University of Vienna, Türkenschanzstrasse 17, 1180 Vienna Austria \\ e-mail: mittermayer@astro.univie.ac.at
}

Received 5 December 2000 / Accepted 15 December 2000

\begin{abstract}
The profiles of $\mathrm{H} \alpha$ in a number of cool Ap stars are anomalous. Broad wings, indicative of temperatures in the range 7000-8000 K end abruptly in narrow cores. The widths of these cores are compatible with those of dwarfs with temperatures of $6000 \mathrm{~K}$ or lower. This profile has been known for Przybylski's star, but it is seen in other cool Ap stars. The $\mathrm{H} \beta$ profile in several of these stars shows a similar core-wing anomaly (CWA). In Przybylski's star, the CWA is probably present at higher Balmer members. We are unable to account for these profiles within the context of LTE and normal dwarf atmospheres. We conclude that the atmospheres of these stars are not "normal". This is contrary to a notion that has long been held.
\end{abstract}

Key words. stars: chemically peculiar - stars: chromospheres - stars: oscillations

\section{Introduction}

It has been widely assumed that the profiles of the Balmer lines in chemically peculiar (CP) stars are normal. This does not mean that small difficulties in getting precise fits do not occur, but that generally speaking, such problems are no more serious for CP stars than those with normal compositions (cf. Barklem et al. 2000).

The strangest of all CP Stars, Przybylski's star (HD 101065), was known to have unusual Balmer profiles. This was shown by Wegner (1976), and Przybylski (1979) himself. Figure 1 was constructed from a table in Przybylski's paper, which appeared in one of the less accessible journals. It illustrates how the core of the $\mathrm{H} \alpha$ profile in HD 101065 is markedly sharper than those of a sample of early to late F-stars. From the wing strengths alone, one might conclude that HD 101065 is a middle

Send offprint requests to: C. R. Cowley, e-mail: cowley@umich.edu

* Based on observations obtained at the European Southern Observatory, Paranal, Chile (ESO programme No. 65.I-0644) and the South African Astronomical Observatory (SAAO).

** Now at ESO, e-mail: shubrig@eso.org
$\mathrm{H} \alpha$ Profiles

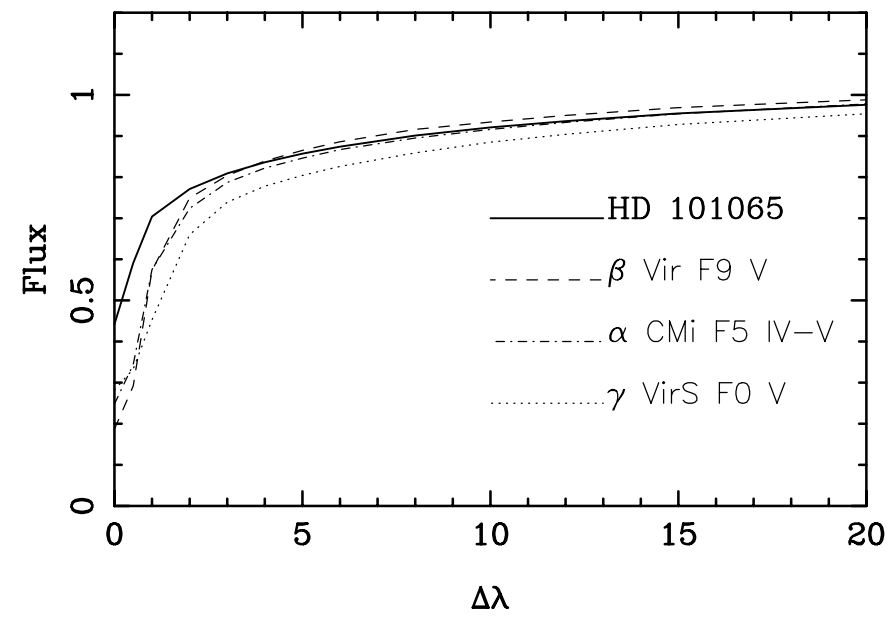

Fig. 1. H $\alpha$ profiles of HD 101065 and 3 F-dwarfs, plotted from data in Przybylski (1979). Though Przybylski's resolution was much lower than that illustrated in Figs. 2, 3, 4, below, it was clear to him that the core-wing structure of HD 101065 was anomalous 


\section{$\mathrm{H} \alpha$ Profiles}

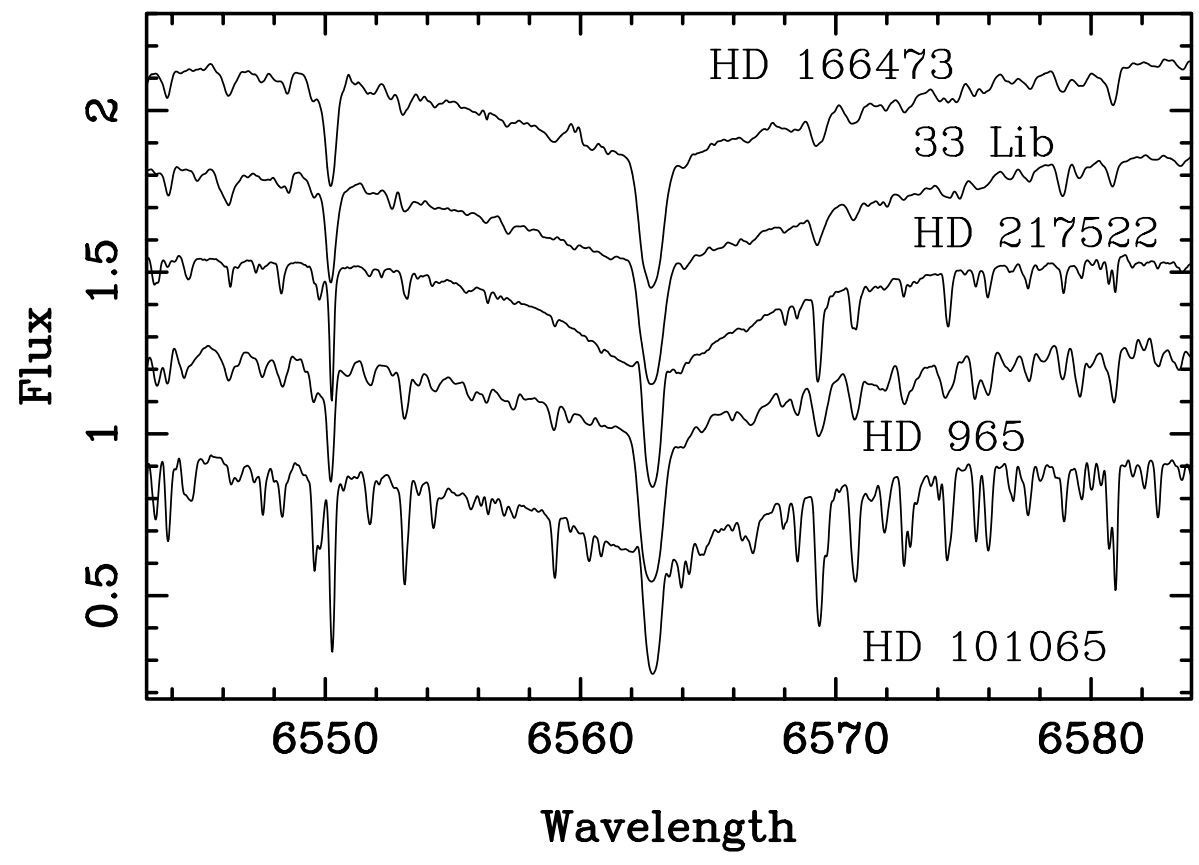

Fig. 2. $\mathrm{H} \alpha$ profiles of 5 cool Ap stars showing the core-wing anomaly (CWA). All spectra above that of HD 101065 have been displaced upward by $0.4,0.6,1.0$, or 1.3 units
F-dwarf. This, indeed, was the conclusion of Cowley et al. (2000, henceforth P1) in a recent abundance analysis. They adopted $T_{\mathrm{e}}=6600 \mathrm{~K}$, and $\log (g)=4.2$, very close to recent tabulations for mid-F dwarfs (cf. Drilling \& Landolt 1999).

The authors of P1 were aware of the unusual nature of the Balmer lines in HD 101065, but had no solution to the riddle of the sharp cores of $\mathrm{H} \alpha$ and $\mathrm{H} \beta$. Their selection of $6600 \mathrm{~K}$ was based primarily on the ionization balance of iron-group spectra. The hydrogen line wings, and the ionization balance of the second and third spectra of neodymium and praseodymium indicated temperatures roughly a thousand degrees higher.

Both Przybylski and Wegner suggested that these profiles might be understood in terms of an atmosphere that was influenced by severe line blanketing. Within the context of an LTE calculation, we have been unable to reproduce both the deep, narrow cores and the wings. Several numerical experiments with blanketed models were investigated in the preparation of P1. Even if the boundary temperature were artificially lowered below the values in the models discussed in that paper, it was not possible to reproduce the narrow, deep $\mathrm{H} \alpha$ core.

\section{Balmer lines of other cool, magnetic CP stars}

The profiles of the Balmer lines of CP stars in the magnetic sequence have not been extensively studied. It is very difficult to reduce high-resolution echelle spectra in such a way that the profiles of the Balmer lines are reliable. These objects are generally more difficult to study than their non-magnetic congeners, the Am and HgMn stars. Their surfaces are known to show spatial inhomogeneities (abundance patches), so the meaning of any traditional abundance study is uncertain. Much of the recent spectroscopic work on these stars has been concerned with spatial mapping of the chemical inhomogeneities, or line identifications.

We have recently examined high resolution spectra of several cool CP stars in the magnetic sequence, and find some have deep cores at $\mathrm{H} \alpha$ and $\mathrm{H} \beta$ similar to those of HD 101065. Figure 2 shows profiles (from top to bottom) of HD 166473 (CoD -37 12303), HD 137949 (33 Lib), HD 217522 (CoD - 45 14901), HD 965 (BD -0 21), and HD 101065 (CoD - 46 5445). Spectra of first two stars were obtained by PM at South Africa Astronomical observatory (SAAO) using the fiber-fed GIRAFFE echelle spectrograph with a resolution of 35000 . These spectra were reduced with the standard IRAF package, but special efforts were taken to make a proper continuum level in echelle orders with hydrogen lines. For the other three stars the spectra were obtained by SH at ESO using the UV-Visual Echelle Spectrograph of the VLT. The original resolution is estimated to be 110000 . The spectra were mildly filtered, and normalized by CRC using Michigan software, but the sharp cores are plainly visible in the unnormalized spectra.

At the moment, it is not known whether HD 965 is a rapidly oscillating Ap star (roAp), but other four stars illustrated are known roAp stars.

A CWA is present in $\mathrm{H} \beta$ for some of the stars of Fig. 2, as shown for one of them in Fig. 3 .

The CWA is often less pronounced at $\mathrm{H} \beta$ than $\mathrm{H} \alpha$. In HD 101065, the anomaly persists into the higher series members, as was noted by Wegner (1976). In HD 217522, 
$\mathrm{H} \boldsymbol{\beta}$ Spectra

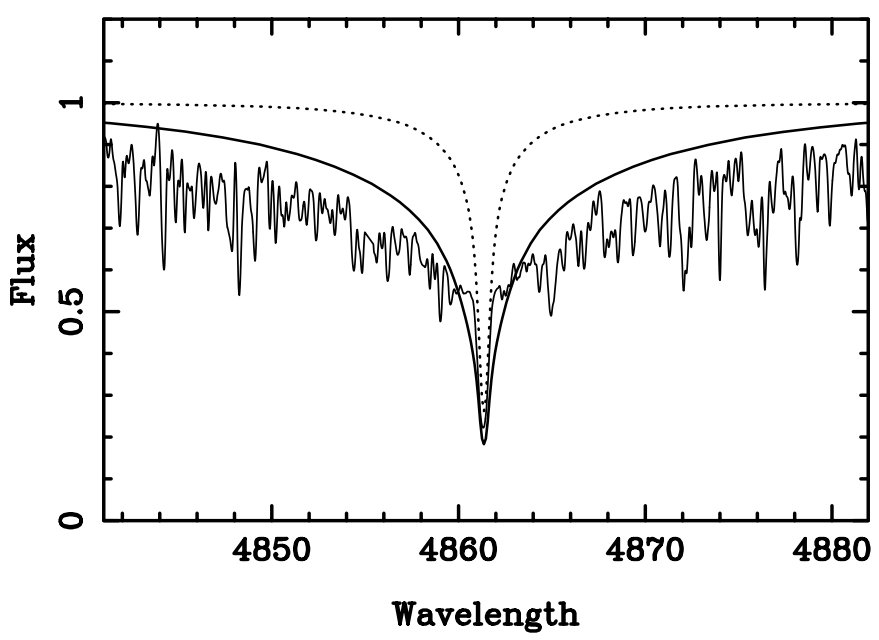

Fig. 3. $\mathrm{H} \beta$ profile in HD 965. The smooth curves are from the Kurucz (1994) library of profiles for normal abundances with $V$ turb $=0$. The dashed curve is for $T_{\mathrm{e}}=5500 \mathrm{~K}$, the solid curve for $T_{\mathrm{e}}=7000 \mathrm{~K}$. Both profiles are for $\log (g)=4.0$. A high point in the stellar spectrum near $\lambda 4855$ caused by hot pixels has been artificially removed

one can see (Fig. 2) at $\mathrm{H} \alpha$, a possible transition case. The slope of the profile steepens toward the core, beginning some 3 to 4 angstroms from the line center. This shape is arguably less noticeable in the other four spectra. $\mathrm{By} \mathrm{H} \beta$, the CWA is hardly noticeable in HD 217522.

van't Veer \& Megessier (1996) studied $\mathrm{H} \alpha$ and $\mathrm{H} \beta$ profiles of the Sun, Procyon, and the cool Am stars 63 Tau and $\tau$ UMa (cf. their Fig. 5). More recently, Gardiner et al. (1999) discussed $\mathrm{H} \alpha$ profiles in a dozen normal stars (including the sun) with effective temperatures between $5777 \mathrm{~K}$ and $9940 \mathrm{~K}$. Their spectra do not have the sharp break in slope that can be seen in our Fig. 2. The recent study of the roAp star HR 3831 (HD 83332) by Baldry \& Bedding (2000) may not have had sufficient resolution to reveal a CWA. We tentatively conclude the phenomenon is limited to the magnetic sequence of $\mathrm{CP}$ stars. It remains to be seen if it is also limited to the roAp stars.

The best studied cool Ap star that is not a roAp, is $\beta$ CrB. This star has similar atmospheric parameters to the roAp star $\gamma$ Equ. The spectrum of $\beta \mathrm{CrB}$ was obtained at McDonald observatory with a resolution of 56000 . We display it in Fig. 4, along with a spectrum of $\gamma$ Equ from the same observational run at SAAO on which the upper two spectra of Fig. 2 were obtained.

We compare the $\mathrm{H} \alpha$ lines for both stars with a theoretical line profile calculated by spectrum synthesis code SYNTH, which incorporates the latest version of hydrogen line broadening (Barklem et al. 2001). The comparison is shown in Fig. 4. Note the following:

- A break in the slope of $\mathrm{H} \alpha$ line profile is similar for both the roAp star $\gamma$ Equ and the non-oscillating star $\beta \mathrm{CrB}$, but it is less extreme than for the stars of Fig. 2;

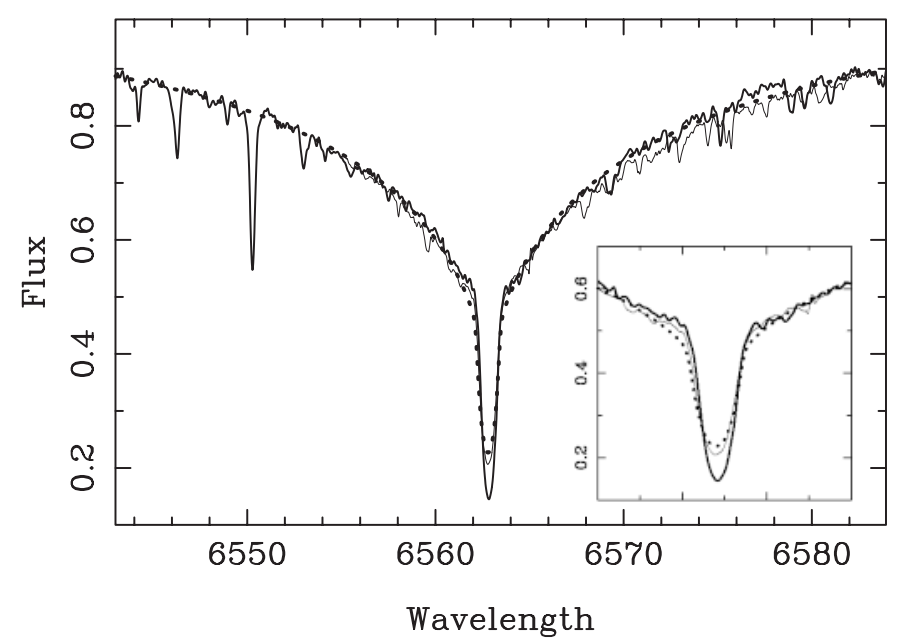

Fig. 4. Observed $\mathrm{H} \alpha$ profiles for $\gamma$ Equ (thick line), $\beta \mathrm{CrB}$, (thin line) and a calculated profile (dotted) for $T_{\mathrm{e}}=7800 \mathrm{~K}$, $\log (g)=4.2$. The inset shows the same data, which has been plotted at a different scale to illustrate the near fit between the theoretical calculations and the observations

- This break is also present on theoretical line profile: the slow decaying wings of a hydrogen line are formed by energy level perturbations by electrons and protons. The quasi-static approximation used to compute the Stark profile predicts the very narrow core. The additional thermal Doppler broadening is virtually unnoticeable everywhere except in the line core. Thus, the core has a Doppler shape reflecting the temperature and velocity field of the top atmospheric layers;

- It is prudent to say that the break in the slope is only, "marginally" different from theoretical predictions.

The stars of Fig. 2 are all cooler than $\beta \mathrm{CrB}$ and $\gamma$ Equ. But at least one cool roAp, HD 122970 has an $\mathrm{H} \alpha$ profile that is well matched by theory. Possibly the roAp phenomenon may be a necessary condition for the CWA, but not a sufficient one.

\section{Interpretation}

We note that a core-wing structure develops in normal dwarfs with temperatures between 7000 and $8000 \mathrm{~K}$. This is illustrated in Fig. 5, with the profiles taken from Kurucz (1994). This structure may account for the reasonable fit shown in Fig. 4. It is not what we are calling the CWA shown in Figs. 2 and 3. Those stars are cooler than $\beta \mathrm{CrB}$ and $\gamma \mathrm{Equ}$, and have temperatures closer to 7000 than $8000 \mathrm{~K}$. Their $\mathrm{H} \alpha$ profiles have a much more abrupt change in the slope between the wing and the core than is seen in normal stars or the calculations of Fig. 5.

Deep cores in an LTE calculation are usually made by a low boundary temperature. In the case of hydrogen, the high-excitation $n=2$ level becomes rapidly depopulated as the temperature drops, so that the cores are relatively 
$\mathrm{H} \alpha$ Spectra

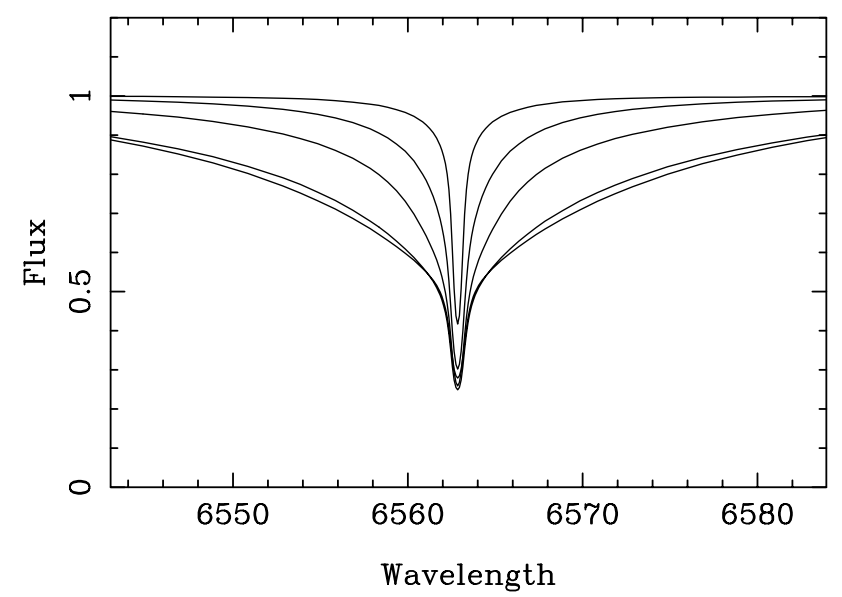

Fig. 5. Calculated $\mathrm{H} \alpha$ profiles for normal dwarfs $(\log (g)=4.0)$ with temperatures of 5000 (weakest line), 6000, 7000, 8000, and $9000 \mathrm{~K}$

insensitive to a lower boundary temperature. We have attempted arbitrarily adding a cool, dense layer to the top of our atmospheres. In LTE, low-excitation metal lines become unrealistically strong well before the $\mathrm{H} \alpha$ core becomes as deep as in the observations.

We have attempted to reproduce the CWA by arbitrary modifications of the temperature distribution that might be attributed to convection, or convection in a magnetic, pulsating atmosphere. These attempts were completely unsuccessful. For the present, we assume that only a fully non-LTE calculation can be expected to reproduce the observed profiles.

We have also performed non-LTE calculations using the MULTI code (Carlsson 1992) for the first three Balmer lines for two model atmospheres with $T_{\text {eff }}=6000$ and $8000 \mathrm{~K}$. The resulting profiles have slightly (less than 5\%) deeper cores with largest effect in $\mathrm{H} \alpha$, but we find no difference in the width of the core. The potential effect of the partial redistribution during scattering in the line cores remains to be investigated.

It now seems plain that the atmospheres of magnetic CP stars cannot be considered normal, even setting aside the well known chemical inhomogeneities or abundance "patches". In addition to the sharp cores of $\mathrm{H} \alpha$ and $\mathrm{H} \beta$, there is the ionization discrepancy between the second and third spectra of the lanthanide rare earths. This problem was discussed by Ryabchikova and her colleagues (cf. Ryabchikova et al. 2000; Gelbmann et al. 2000), and also in P1. Essentially, abundances from the third spectra, primarily of Pr III and Nd III are an order of magnitude greater than those of the second spectra of these elements.

It is unlikely the ionization anomaly can be due to the neglect of hyperfine structure in Pr III or odd-N isotopes of Nd III, because the lines of these spectra are relatively weak in some of the roAp stars (cf. Ryabchikova et al. 2000, HD 122970 and $10 \mathrm{Aql}$ ). Moreover, the same ions can be observed in non-oscillating Ap stars and do not show the same anomaly (Ryabchikova et al. 2001; Weiss et al. 2000).

Our partial analysis of HD 965 suggests that the same kind of ionization anomaly exists between Fe I and II. The effective temperature of a model which makes the abundances from Fe I and II lines equal is 8000-9000 K, while the color temperature of the star is in the range 7000-7500 K. A low surface gravity palliates this somewhat, but no realistic gravity will reconcile the ionization and color temperatures.

We are left with distinct indications of two temperatures in models whose structures are quite uncertain. The core depth of the early Balmer lines argues for their formation in a region that covers most of the star. If, indeed, a high layer of gas overlies the photospheres of these stars as in the model by Babel (1992), it could play an important role in the origin of the chemical anomalies of these magnetic Ap stars.

Acknowledgements. CRC thanks many colleagues for helpful conversations regarding HD 965 and the CWA. He thanks Dr. Saul J. Adelman for sending Balmer profiles of several cool Ap stars. Thanks are due to C. van't Veer-Menneret, B. Smalley, and R. Gardiner for commenting on profiles of AmFm and normal stars.

Research at the Institute for Astronomy in Vienna received funding from the Austrian Fonds zur Förderung der wissenschaftlichen Forschung (Project S7303-AST).

\section{References}

Babel, J. 1992, A\&A, 258, 449

Baldry, I. K., \& Bedding, T. R. 2000, MNRAS, 318, 341

Barklem, P. S., Piskunov, N., \& O'Mara, B. J. 2000, A\&A, 355, L5

Barklem, P. S., Piskunov, N., \& O'Mara, B. J. 2001, A\&A, in press

Carlsson, M. 1992, in Cools Stars, Stellar Systems and the Sun, proc. of the 7th Cambridge workshop (ASP: San Francisco), vol. 26, 499

Cowley, C. R., Ryabchikova, T. A., Kupka, F., et al. 2000, MNRAS, 317, 299

Drilling, J. S., \& Landolt, A. U. 1999, in Allen's Astrophysical Quantities, 4th edition, ed. A. N. Cox (Springer Verlag)

Gardiner, R. B., Kupka, F., \& Smalley, B. 1999, A\&A, 347, 876

Gelbmann, M., Ryabchikova, T., Weiss, W. W., et al. 2000, A\&A, 356, 200

Kurucz, R. L. 1994, CD-Rom No. 19 (Smithsonian Ap. Observatory)

Przybylski, A. 1979, New Zealand J. Sci., 22, 429

Ryabchikova, T. A., Savanov, I. S., Hatzes, A. P., Weiss, W. W., \& Handler, G. 2000, Astron. Ap., 357, 981

Ryabchikova, T. A., Savanov, I. S., Malanushenko, V. P., \& Kudryavtsev, D. O. 2001, Astron. Rep., 78, in press

van't Veer-Menneret, C., \& Mégessier, C. 1996, A\&A, 309, 879

Wegner, G. 1976, MNRAS, 177, 99

Weiss, W. W., Ryabchikova, T. A., Kupka, F., et al. 2000, in The Impact of Large-Scale Surveys on Pulsating Star Research, ASP Conf. Ser., 203, 487 